\section{NEMVALEUKIN ALFA, A NOVEL ENGINEERED IL-2 CYTOKINE, IN COMBINATION WITH THE ANTI-PD-1 ANTIBODY PEMBROLIZUMAB IN PATIENTS WITH RECURRENT/METASTATIC HEAD AND NECK SQUAMOUS CELL CARCINOMA (ION-01 STUDY)}

${ }^{1}$ Brian Gastman*, ${ }^{2}$ Mac Cheever, ${ }^{2}$ Steven Fling, ${ }^{3}$ Cesar Perez, ${ }^{4}$ Manish Patel, ${ }^{5}$ Jessica Geiger, ${ }^{6}$ Zujun Li, ${ }^{7}$ Marshall Posner, ${ }^{8}$ Conor Steuer, ${ }^{2}$ Leonard D'Amico, ${ }^{2}$ Angela Shaulov Kask, ${ }^{9}$ Yangchun Du, ${ }^{9}$ Derek Matthies, ${ }^{9}$ Sung Jin Huh, ${ }^{9}$ Yan Wang, ${ }^{9}$ Julie Graham, ${ }^{10}$ Laura Chow. ${ }^{1}$ Cleveland Clinic, Cleveland, OH, USA; ${ }^{2}$ Fred Hutchinson Cancer Research Center, Seattle, WA, USA; ${ }^{3}$ Sylvester Comprehensive Cancer Center, Miami, FL, USA; ${ }^{4}$ Masonic Cancer Center, Rochester, MN, USA; ${ }^{5}$ Cleveland Clinic Main Campus, Cleveland, OH, USA; ${ }^{6}$ Perlmutter Cancer Center at NYU, New York, NY, USA; ${ }^{7}$ Icahn School of Medicine at Mt Sinai, New York, NY, USA; ${ }^{8}$ Winship Cancer Institute, Atlanta, GA, USA; ${ }^{9}$ Alkermes, Inc., Waltham, MA, USA; ${ }^{10}$ University of Texas Austin, Austin, TX, USA

Background Nemvaleukin alfa (nemvaleukin, ALKS 4230) is a novel engineered cytokine that selectively binds to the intermediate-affinity IL-2R to preferentially activate and expand anti-tumor CD8 $+\mathrm{T}$ and $\mathrm{NK}$ cells with minimal expansion of regulatory $\mathrm{T}$ cells (Treg), thereby leveraging antitumor effects of the IL-2 pathway while mitigating potential toxicity that limits use. ${ }^{1}$ Nemvaleukin single-agent activity has been demonstrated in checkpoint inhibitor-experienced patients, and deep and durable responses have been achieved in combination with pembrolizumab in multiple tumor types (eg, breast, head and neck, gastrointestinal, genitourinary, gynecological). ${ }^{2}$

Methods ION-01 (NCT04144517) is a nonrandomized trial in adult patients with histologically/cytopathologically confirmed diagnosis of metastatic/recurrent head and neck squamous cell carcinoma. Eligible patients have progressive disease after $\geq 8$ weeks on anti-PD-(L)1 therapy. The primary endpoint is the rate of new or improved antitumor response after the addition of nemvaleukin. Secondary objectives include characterization of the antitumor response and evaluation of safety and tolerability of the combination regimen. Patients receive intravenous nemvaleukin ( $3 \mu \mathrm{g} / \mathrm{kg}$ ) once daily for the first 5 days and pembrolizumab $(200 \mathrm{mg})$ on day 1 of each 21 -day cycle. Tumor imaging and biopsies were performed at baseline and at pre-specified times. We present preliminary safety and antitumor activity (RECIST v1.1) data as of June 2021.

Results Fourteen patients with progressive disease received combination therapy with nemvaleukin and pembrolizumab; 8 had no prior response to pembrolizumab, 6 had previous best response of stable disease or partial response. Mean ( \pm SD) age was $62 \pm 12$ years, $86 \%$ were male, and all were Caucasian. Prior anti-cancer therapy included radiotherapy (93\%) and surgery (50\%). ECOG performance status was $0(14 \%)$ and $1(86 \%)$ at baseline. Treatment-related adverse events of any grade in $\geq 30 \%$ of patients were chills (64.3\%), pyrexia $(57.1 \%)$, fatigue $(42.9 \%)$, and nausea $(35.7 \%)$. Five patients had stable disease as best response. One patient achieved a partial response (complete response in the target lesion) and remains on treatment $(8+$ cycles $)$. Expansion of CD $8+\mathrm{T}$ and NK cells with minimal Treg expansion was observed.

Conclusions Nemvaleukin and pembrolizumab combination therapy was generally well tolerated; adverse events were consistent with those observed with intravenous nemvaleukin in ARTISTRY studies [2]. Peripheral immune cell expansion profiles are comparable to that observed with the same regimen in the ARTISTRY 1 phase 1 study. Emerging data from pretreatment and on-treatment paired biopsies will further characterize specific antitumor effects of nemvaleukin and pembrolizumab in this patient population.
Acknowledgements The authors would like to thank all the patients who are participating in this study and their families. The study is sponsored by Alkermes, Inc. Medical writing and editorial support was provided by Parexel, and funded by Alkermes, Inc.

Trial Registration ClinicalTrials.gov NCT04144517

\section{REFERENCES}

1. Lopes JE, Fisher JL, Flick HL, et al. ALKS 4230: a novel engineered IL-2 fusion protein with an improved cellular selectivity profile for cancer immunotherapy. Immunother Cancer 2020;8:e000673. doi: 10.1136/jitc-2020-000673.

2. Boni $V$, Winer IS, Gilbert $L$, et al. ARTISTRY-1: Nemvaleukin alfa monotherapy and in combination with pembrolizumab in patients (pts) with advanced solid tumors. J Clin Oncol 2021;39(Suppl 15):abstr 2513.

Ethics Approval This study was approved by Quorum Review IRB (now Advarra IRB), approval number QR 33752.

http://dx.doi.org/10.1136/jitc-2021-SITC2021.432 